\title{
Kernos
}

Revue internationale et pluridisciplinaire de religion grecque antique

6 | 1993

Varia

\section{Les indices archéologiques du sacrifice humain grec en question}

Compléments à une publication récente

\section{Pierre Bonnechere}

\section{(2) OpenEdition \\ 1 Journals}

\section{Édition électronique}

URL : http://journals.openedition.org/kernos/534

DOI : $10.4000 /$ kernos.534

ISSN : 2034-7871

Éditeur

Centre international d'étude de la religion grecque antique

\section{Édition imprimée}

Date de publication : 1 janvier 1993

Pagination : 23-55

ISSN : 0776-3824

\section{Référence électronique}

Pierre Bonnechere, «Les indices archéologiques du sacrifice humain grec en question », Kernos [En ligne], 6 | 1993, mis en ligne le 07 avril 2011, consulté le 01 mai 2019. URL : http:// journals.openedition.org/kernos/534; DOI : 10.4000/kernos.534 
Kernos, 6 (1993), p. 23-55.

\section{LES INDICES ARCHÉOLOGIQUES}

\section{DU SACRIFICE HUMAIN GREC EN QUESTION :}

\section{COMPLÉMENTS À UNE PUBLICATION RÉCENTE}

Septante-cinq années ont été nécessaires pour que la discussion sur le sacrifice humain grec soit véritablement rouverte. Dennis Hughes, dans la publication de sa thèse de doctorat, intitulée Human Sacrifice in Ancient Greece, parue en 1991 chez Routledge (Londres et New York), s'est ainsi lancé sur les traces de Friedrich Schwenn (Die Menschenopfer bei den Griechen und Römern, Giessen, 1915 [RGVV, 15.3]), dont le travail solide était demeuré l'unique référence à propos de ce sombre sujet. J'ai fait ailleurs le compte rendu de cet excellent ouvrage, mais j'aimerais ici apporter quelques précisions, quelques additions, aux chapitres consacrés à l'archéologie, ainsi que militer en faveur du respect des plus élémentaires principes de critique historique et, bien que n'étant pas archéologue de formation, de déontologie archéologique*.

* Si le hasard fait parfois mal les choses, puisque Dennis Hughes m'a devancé de peu dans la publication de sa thèse, il a réparé son injustice en cette occasion car les perspectives de cet auteur et les miennes sont, pour l'essentiel, différentes et complémentaires: l'essentiel de mes recherches a porté en effet sur les fondements initiatiques des légendes de sacrifice humain, aussi partie prenante de la mythologie des fêtes de renouvellement, ainsi que sur l'impact du thème dans la mentalité des Grecs, aspects signalés mais peu abordés par notre collègue américain: aussi l'essentiel de mon travail sera-t-il publié, tandis que les chapitres archéologiques, faisant double emploi avec les siens, sont ici transposés dans les seuls éléments qui me semblent pouvoir apporter quelque chose de neuf. Sur l'ensemble du livre de Hughes, voir mon compte rendu dans $A C$, à paraître. 


\section{Anemospilia (Archanes, Crète) : un sacrifice humain en temps de crise?}

Dennis Hughes ouvre sa recension archéologique par l'analyse de la découverte retentissante des époux Sakellarakis à Anemospilia sur le Mont Jouktas, en 1979, et dont l'interprétation ambitionnait, en quelque sorte, de fournir une preuve archéologique du sacrifice humain en Crète. Rappelons qu'à partir des résultats de fouilles soignées, les inventeurs en étaient arrivés à cette conclusion : vers 1700 avant J.-C., lors d'un tremblement de terre qui secouait la montagne, un prêtre et une acolyte auraient, dans la chambre ouest du temple élevé au flanc de la colline, procédé à l'immolation d'un jeune homme d'environ 18 ans, renversé pieds et mains liés sur une table sacrificielle. Le coup fatal aurait été porté à l'aide d'une lame de bronze, une $\mu \alpha \chi \chi a i \rho a ~ d e ~ q u a r a n t e$ centimètres abandonnée sur le corps sans vie du sacrifié. Le toit ébranlé de l'édifice s'effondra à ce moment sur les protagonistes du drame, gelant dans le temps cette sinistre cérémonie 1 .

Les Sakellarakis s'étaient efforcés d'établir une relation directe entre leurs trouvailles et la pratique des sacrifices animaux dans le culte local : pour eux le bâtiment est un temple, la chambre ouest est le siège habituel des sacrifices et le couteau trouvé est une $\mu \alpha ́ x a t p a$. Hughes a montré à souhait que cet effort pouvait être réduit à néant ${ }^{2}$ : certes le bâtiment, si sa fonction religieuse est certaine, n'est pas obligatoirement un temple ou un sanctuaire; certes la chambre ouest, lieu du prétendu sacrifice, n'a pas pu servir d'emplacement des sacrifices ordinaires, puisque cela aurait nécessité le passage de gros animaux au travers d'un corridor encombré de vaisselle précieuse, et surtout au travers d'une porte dont l'ouverture ne doit guère dépasser le mètre de largeur; certes la plate-forme dite sacrificielle ne ressemble-t-elle guère aux autels de bois connus essentiellement par les sources figurées, tel celui représenté sur le sarcophage d'Aghia Triadha; certes

1 Rapports préliminaires mais détaillés des fouilles : Y. et E. SAKELLARAKIS,

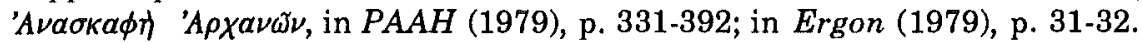
L'article intitulé Drama of Death in a Minoan Temple, paru dans le National Geographic (159, 1981, p. 205-221), est certes plus accessible mais beaucoup plus allusif. C'est cependant là qu'on trouvera énoncée l'hypothèse du sacrifice humain avec le plus de détails. Voir encore H. Catling, Archaeology in Greece, 1979-80, in AR (1979-1980), p. 50-51; ID., Archaeology in Greece, 1980 81 , in $A R$ (1980-1981), p. 42.

2 Human Sacrifice, p. 15-17, 47 et 254, n. 24. 
enfin la « $\mu \alpha \chi \alpha \iota \rho \alpha »$ n'en est pas nécessairement une, et sa forme pourrait éventuellement l'apparenter davantage à une pointe de lance qu'à une épée.

Cependant l'analyse des inventeurs, ainsi que les critiques de cette analyse énoncées par Hughes, concernent un faux problème, en ce qu'elles ramènent l'ensemble des données à la quotidienneté, et font assez peu de cas des circonstances exceptionnelles : ne puis-je pas supposer en toute bonne foi que des fonctionnaires - religieux (?) - aient pu sacrifier un homme dans une des chambres d'un édifice dont la vocation religieuse est incontestable, - même si l'appellation de temple reste prématurée, - sur une élévation reconvertie à la hâte en autel de fortune, avec une pointe de lance en guise de couteau à égorger ? Puisque l'immolation humaine apparaît comme une tentative désespérée pour enrayer la progression du cataclysme, tous les efforts accomplis afin de trouver dans les sources archéologiques les traces du comportement sacrificiel traditionnel sont irrémédiablement voués à l'échec : les circonstances extrêmes ont souvent des conséquences inattendues desquelles s'accommodent mal les raisonnements qui accordent trop d'importance à la logique. Aussi ces points disputés de la nature de l'édifice et de la lame de bronze n'entrent-ils que très secondairement en ligne de compte pour conforter ou pour contredire l'hypothèse du sacrifice humain.

Cette dernière se fonde essentiellement sur la position du squelette du "sacrifié" et sur la situation de la lame de bronze qui reposait sur celui-ci. Les coïncidences trompeuses alléguées par Hughes n'ont malheureusement pas plus de poids que l'option sacrificielle préconisée par les fouilleurs. Je signale cependant que l'examen des photographies $^{3}$ n'interdit pas de concevoir que l'homme qu'on dit avoir été sacrifié ait lui-même tenu la lame qui fut retrouvée sur son squelette, fût-il animé ou non d'intentions agressives, - et ce même si ce dernier avait les mains ramenées contre la poitrine : il aurait fort bien pu lâcher l'arme au moment du drame afin de se protéger. À la limite, s'il s'agissait d'une épée, celle-ci aurait même pu être attachée à sa ceinture et avoir été retournée vers le haut à la suite d'un mouvement maladroit lors de l'ensevelissement soudain.

La principale faille de l'hypothèse des Sakellarakis, en fait, réside dans leur explication de la coloration qu'acquirent les os de la "victime" lors de leur crémation : dans la partie gauche du corps, c'est- 
à-dire celle qui se trouvait vers le haut et aurait été vidée de son sang par l'hémorragie consécutive à l'égorgement, les ossements auraient acquis une coloration blanche, tandis que ceux de la partie droite, encore au contact du sang non expulsé par la blessure, suite à l'arrêt cardiaque, auraient viré au noir. Cette affirmation, comme l'a souligné Hughes, est sans fondement, car la coloration des os ne dépend nullement de la présence de sang dans un cadavre livré au feu mais bien de l'intensité calorifique du brasier, laquelle peut varier considérablement dans une même pièce.

Cependant, le rejet de cet argument est plus grave qu'il n'y paraît : l'important, en effet, est de remarquer que les archéologues ne sont plus partis du constat de base pour inférer une conclusion; au contraire, ils ont emprunté le chemin inverse : étant donné qu'aucune analyse scientifique stricte du phénomène n'aurait pu aboutir à une telle affirmation, c'est bien leur théorie du sacrifice qui les a influencés dans l'explication de la coloration étrange des os de celui qui était pour eux un sacrifié. Or, dès que disparaît l'argument fondé sur la coloration des ossements, l'hypothèse est privée de son seul indice vraiment déterminant, car lui seul pouvait permettre de conclure à la mort du jeune homme suite à une hémorragie, mort par conséquent antérieure à la destruction de l'édifice et à la crémation de la dépouille. À partir du moment où cette antériorité n'apparaît plus décisive, ou même seulement probable, la thèse sacrificielle perd toute valeur prédominante. Notons enfin que même si la mort par hémorragie avait pu être par ce biais démontrée, celle-ci aurait pu être accidentelle : l'effondrement de la toiture aurait ainsi pu blesser mortellement le jeune homme, qui serait décédé avant que le bâtiment ébranlé ne prenne feu.

En conclusion, l'hypothèse des Sakellarakis est des plus fragiles : aussi se limitera-t-on à soutenir que les trois personnes qui se trouvaient dans la chambre ouest, dont une portait une épée, furent ensevelies simultanément quand la toiture vint se fracasser sur le sol. Celles-ci trouvèrent la mort, lors d'un séisme, en des circonstances qu'il n'est pas possible d'élucider totalement, mais qui ne laissent à la thèse d'un sacrifice humain qu'une place plus que menue dans l'éventail des possibilités. Et il n'existe, au demeurant, aucune raison de favoriser cette thèse vis-à-vis de celles qui, de façon moins extraordinaire mais plus plausible, envisagent le décès accidentel des trois personnes incriminées. 
Rien n'autorise davantage les Sakellarakis à établir un rapprochement entre le cas d'Anemospilia, marqué par l'imminence du désastre, et les légendes grecques de sacrifices humains accomplis lors des périodes de crise : pour qu'un tel rapprochement soit fondé, il faudrait d'abord établir avec certitude que ces légendes renferment un substrat historique, et qu'elles ne résultent pas d'un processus de création mythique destiné à pourvoir d'une justification «historique» les rumeurs de sacrifices humains qui circulaient dans la Grèce classique à propos des temps héroïques. Or une étude serrée des textes semble plutôt devoir faire pencher la balance de ce côtét.

La réticence provoquée par l'interprétation des inventeurs aux yeux du monde scientifique, toutefois, ne doit pas en elle-même être utilisée comme un argument en défaveur de l'hypothèse du sacrifice : cette réticence doit être tempérée quand on sait l'opposition systématique et presque naturelle à toute allusion de sacrifice humain en Grèce.

Quoi qu'il en soit, les tenants d'un sacrifice humain à Anemospilia devront asseoir leurs positions sur davantage de preuves et moins sur une apparente convergence d'hypothèses. De plus, à supposer qu'immolation humaine il y ait eu, la portée de cette découverte resterait limitée, c'est-à-dire qu'en aucun cas elle ne pourrait autoriser à une généralisation à propos de la pratique des immolations humaines dans le culte minoen, même lors des situations d'extrême détresse : il aurait pu s'agir ici d'une solution ultime, mais qui pourra affirmer que d'autres prêtres auraient ou ont réagi de la sorte? Cette décision ressort avant tout de la psychologie personnelle de l'individu, et sans doute encore davantage du contrôle de la peur, mue par des réactions avant tout instinctives qui peuvent générer des comportements surprenants. Il est important de le signaler, d'autant plus que certains ont déjà inféré, à partir de l'hypothèse des Sakellarakis, que le sacrifice humain pouvait faire partie intégrante du système religieux minoen ${ }^{5}$ : c'est non seulement prématuré, mais sans guère de doute totalement erroné.

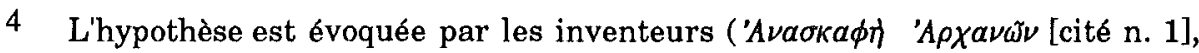
p. 389, n. 1 et 2, d'après les avis de N. Platon et M.P. N ILsson et dans Drama of Death [cité n. 1], p. 218). Sur ces problèmes, voir ma thèse, sous presse; au reste, il n'existe à ma connaissance aucune légende qui mette en scène un sacrifice humain en relation avec un séisme.

5 Ainsi R. Castleden, The Knossos Labyrinth. A New View of the "Palace of Minos" at Knossos, Londres-New York, 1990, p. 122. 


\section{Cnossos, North House (à l'ouest du Stratigraphical Museum) : des sacrifices humains à des fins cannibales ?}

Hughes poursuit son itinéraire archéologique en faisant un détour par Cnossos, où Peter Warren, dès 1979, lors de sa campagne de fouilles à proximité du Stratigraphical Museum (campagne prolongée par celles de 1980 et 1981), prétendait avoir trouvé une trace archéologique de pratiques cannibales dans un culte extatique du Minoen récent I B : la cave d'une maison, la North House, dénommée "Room of the Children's Bones" y avait en effet livré quelque 371 os ou fragments d'os ayant appartenu à un minimum de quatre enfants impubères, ainsi que 251 os d'animaux, ces derniers étant pour les uns cuits, pour les autres non. Plusieurs autres ossements enfantins et quelques ossements d'adultes avaient également été mis au jour dans d'autres pièces de la maison, y compris dans celle appelée "Room of the Frescoes". Les ossements humains, éclatés et éparpillés, n'avaient pas été cuits et se trouvaient donc in situ, dans le sol, lorsque la maison fut ravagée par un incendie qui provoqua la chute du toit. Parmi les ossements animaux, nonante avaient été entaillés, comme l'avaient été, essentiellement à l'emplacement des tendons, environ $36 \%$ des os d'enfants du Room of the Children's Bones et à peu près $16 \%$ de ceux trouvés en dehors de cette pièce. Selon Warren, ces marques, tant sur les ossements animaux qu'humains, témoignent d'un prélèvement intentionnel de la chair à des fins rituelles de manducation ${ }^{6}$.

Dennis Hughes a bien mis en évidence que «everything in the published reports is consistent with a hypothesis that the skeletons were already largely devoid of flesh at the time they were moved into the

6 P.M. W ARREN, Knossos : Stratigraphical Museum Excavations, 1978-80, Part 1, in $A R$ (1980-1981), p. 73-92; ID., Minoan Crete and Ecstatic Religion. Preliminary Observations on the 1979 Excavations in Knossos, [incluant] Postscript on the 1980 Excavations at Knossos, in Sanctuaries and Cults in the Aegean Bronze Age, éds R. HÄGG et N. MARINatos, Stockholm, 1981 (ActaAth, 28), p. 155-166; ID., Knossos. New Excavations and Discoveries, in Archaeology, 37 (1984), p. 48-55; S.M. WALL, J.H. MUSGRAVE et P.M. W ARREN, Human Bones from a Late Minoan I B House at Knossos, in ABSA, 81 (1986), p. 333-388. Cette dernière publication, qui est le rapport détaillé des fouilles, devra seule être consultée à l'avenir en ce qui concerne les découvertes et leur analyse immédiate. Les articles précédents gardent surtout leur valeur en ce qui concerne l'interprétation des résultats, à peine abordée lors de la publication finale (p. 386-388). 
room» ${ }^{7}$ : en fait, seuls certains ossements sont affectés de coupures, et les fouilleurs ont été incapables de reconstituer des squelettes complets. La conclusion qui semble s'imposer est donc que des squelettes, ou des parties de squelettes, déjà décharnés ont été ramenés dans la pièce.

La solution envisagée par Hughes, qui pense à une cérémonie de préparation à l'inhumation finale, coutume que l'on sait en pratique chez les Minoens, est ingénieuse : les ossements proviendraient d'une nécropole; une fois déposés dans la maison de Cnossos, ils auraient été soumis à un dernier nettoyage, qui consistait à enlever les lambeaux de chair encore attenant aux os, précision qui expliquerait pourquoi certains os n'auraient pas été entaillés, ou alors seulement à certains endroits et non pas systématiquement à l'emplacement de tous les tendons (Hughes souligne aussi à bon escient que le prélèvement des chairs n'implique aucunement l'anthropophagie!). Cette solution expliquerait également l'absence caractérisée de certains ossements : lors du transport des dépouilles déjà en partie réduites à l'état de squelette, les os les plus petits, par exemple, et dont on n'a pas trouvé beaucoup de traces, auraient été perdus. Une fois nettoyés, ces os auraient ensuite été destinés à être rapportés dans la nécropole pour l'inhumation seconde et définitive ${ }^{8}$.

Cette solution, dont l'auteur reconnaît le caractère hypothétique, appelle peut-être quelques corrections. Hughes suggère que le principal obstacle à sa théorie repose dans le fait que la maison de Cnossos n'est pas située en contexte funéraire ${ }^{9}$, et Warren avait déjà exploité cet argument pour nier toute possibilité de pratiques d'inhumation ${ }^{10}$. Cependant la nature du quartier reste discutée et tout n'est pas fouillé à $l^{\prime}$ entour ${ }^{11}$ : déclarer sans plus que le contexte n'est pas funéraire reste donc, jusqu'à nouvel ordre, inacceptable.

7 Human Sacrifice, p. 21.

8 Human Sacrifice, p. 21-23. Hughes fait également remarquer que la présence d'ossements animaux est normale, car des restes d'animaux sacrifiés - et donc crus - devant les tombes, ainsi que ceux d'animaux mangés lors des repas funéraires - et donc cuits - avaient pu être ramenés, inopinément en fonction de leur ressemblance avec les restes humains, avec les squelettes d'enfants et d'adultes.

9 Human Sacrifice, p. 23.

10 Knossos : Stratigraphical Museum Excavations (cité n. 6), p. 158.

11 G.C. Geselu, Town, Palace and House Cult in Minoan Crete, Göteborg, 1985 (SIMA, 77), p. 32. 
C'est donc de la disposition et de l'état des ossements qu'il faut tirer quelque élément déterminant. En ce qui concerne les coupures, il est exact qu'elles ressemblent à s'y méprendre à celles qui affectent les ossements du cimetière de Manika, en Eubée, ainsi que Hughes l'a signalé. Une publication préliminaire détaillée de ces ossements, inconnue de ce dernier, précise même, et ce n'est pas une des moindres contradictions fournies par les archéologues en matière de sacrifice humain, que ces marques sont étrangères à tout contexte d'anthropophagie $^{12}$...

Si l'on poursuit les déductions de Peter Warren, et si donc les coupures des ossements de Cnossos étaient vraiment la trace d'un cannibalisme, il faudrait en déduire, avec un cynisme teinté d'humour noir, que respectivement pour le Room of the Children's Bones et l'extérieur de cette chambre, seulement $36 \%$ et $16 \%$ de la chair disponible avaient été retirés de la charpente osseuse, étant donné que le reste des os ne portait pas de marques. Si Hughes a souligné aussi que certains ossements affectés de coupures étaient "of seemingly little culinary appeal» ${ }^{13}$, je soulignerai la difficulté, en cas de dépeçage préalable à la cuisson comme le voudrait Warren, de parvenir à retirer la chair de phalanges des mains et des pieds d'enfants dont le plus âgé n'avait qu'une dizaine d'années. Au reste, puisque les corps n'étaient pas cuits, il faut en inférer que chaque ossement, avant ou après son dépècement, était arraché au squelette, acte dont plus d'un légiste m'a affirmé la difficulté et auquel on trouvera difficilement une explication.

Il est toutefois un élément embarrassant dans l'hypothèse d'une maison funéraire où l'on aurait préparé les squelettes pour leur

12 Voir M. FounToulaKIs, Some Unusual Burial Practices in the Early Helladic Necropolis of Manika, in Thanatos. Les coutumes funéraires en Egée à l'Age du Bronze. Actes du Colloque de Liège (21-23 avril 1986), éd. R. LAFFINEUR, Liège, 1987 (Aegaeum, 1), p. 29-33. La comparaison des entailles sur les ossements de Knossos et ceux de Manica laisse apparaître une grande similitude : de part et d'autre, elles sont nettes, de forme variable, profondes de quatre ou cinq millimètres; elles ont été réalisées par un main connaisseuse de l'anatomie humaine, et apparaisent également sur des os en soi peu susceptibles d'en recevoir : métatarsien, métacarpien, rotule. De plus, des ossements animaux, cuits et non cuits, ont été retrouvés, sur le site eubéen, avec les ossements humains entaillés : il n'y a rien à en déduire pour le moment, mais il faut souligner que les caractéristiques du "festin cannibalesque» de Cnossos existent ailleurs, et cette fois en un contexte on ne peut moins sacrificiel.

13 Human Sacrifice, p. 22. 
dernière inhumation, c'est le fracassement des os : on pourrait comprendre que les squelettes, transbahutés sans trop de précaution, aient pu se disloquer, mais le fait est que la plupart des fragments d'ossements n'excèdent pas quelques centimètres. De la sorte, un calcul approximatif montre que sur environ cent cinquante côtes fragmentaires, un sixième seulement dépasse les nonante millimètres, tandis que la moyenne de toutes les longueurs conservées est d'à peu près cinq centimètres et demi. Pour les fémurs, sur un total de treize fragments, deux seulement sont presque entiers et ainsi de suite ${ }^{14} \ldots$

À l'éclatement s'ajoute la dispersion de ces ossements non seulement dans toutes les pièces de la maison, mais encore à l'intérieur même de chaque chambre : ainsi le crâne catalogué A n'a pu être reconstitué qu'à partir de fragments trouvés à onze endroits différents dans le Room of the Children's Bones, et à neuf endroits pour le crâne $B^{15} \ldots$

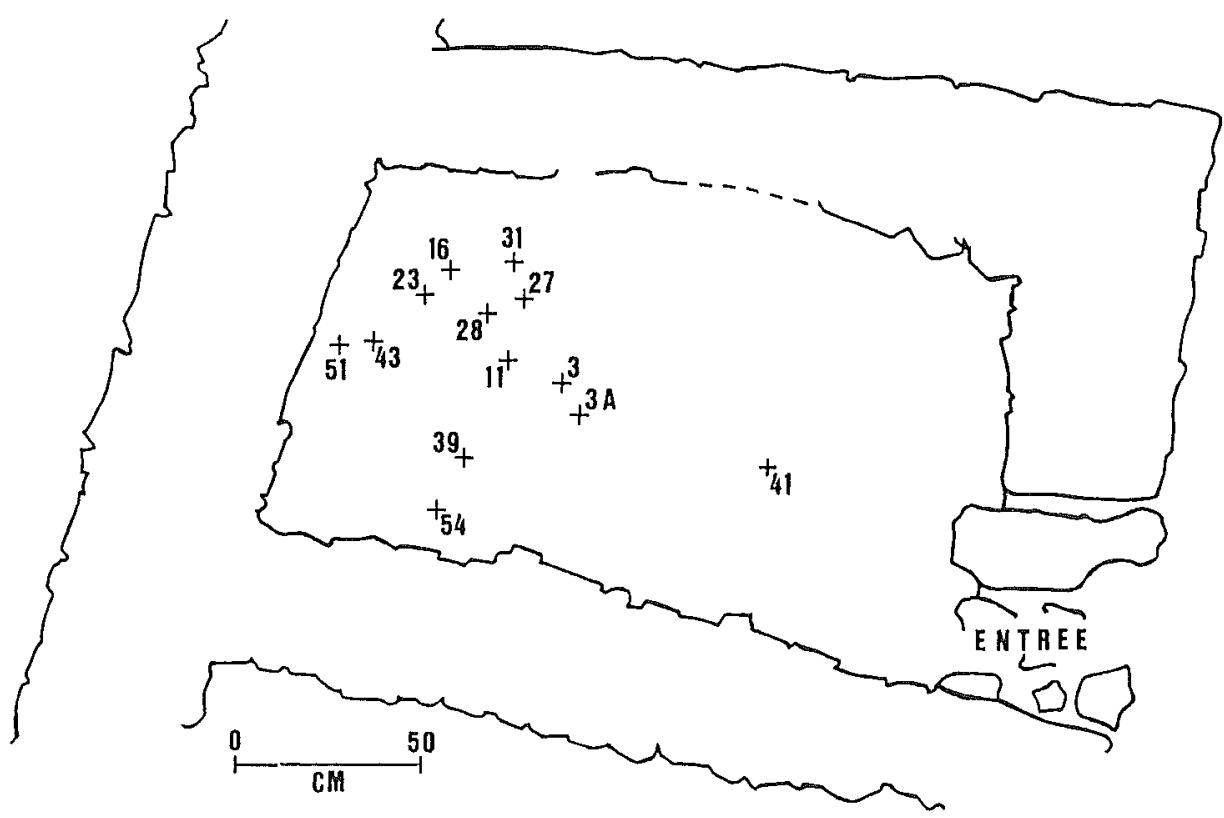

Figure 1 : Cnossos, Room of the Children's Bones. Position in situ des fragments du crâne A, selon la numérotation de Peter Warren (voir n. 14)

14 Voir le tableau apud S.M. Wall, J.H. Musgrave et P.M. WARREN, Human Bones (cité n. 6), p. 349-363.

15 Voir le tableau cité n. 14, $s u b n^{\circ} 1-2$ (p. 349). 
Mais si cette dispersion des vestiges humains semble peu en accord avec une seconde cérémonie funéraire pratiquée dans la maison de Cnossos, il est très probable, en revanche, que les entailles réalisées au couteau soient effectivement les traces d'une opération post mortem : je considérerais volontiers que cette cérémonie avait déjà été accomplie, préalablement ou non à l'ensevelissement définitif, mais ailleurs et surtout avant le transport des ossements dans la maison cnossienne. Le fracassement des ossements ne m'est connu, mais sans doute en existet-il d'autres exemples, que dans les usages funéraires des utilisateurs des tombes à chambre de la Mésara ${ }^{16}$.

Aussi, plutôt que de soutenir l'hypothèse selon laquelle la maison de Cnossos était une sorte de chapelle funéraire, je propose deux autres embryons de solution :

- soit les ossements n'ont ici aucune fonction particulière : leur présence serait simplement due à l'utilisation de terre de remblai prélevée dans un site funéraire ancien et oublié. Serait expliquée de la sorte la présence tant d'ossements issus des mêmes squelettes que l'absence des os de petite taille ou de conservation fragile. La découverte, sur le site, d'ossements d'adultes n'aurait rien d'exceptionnel dans cette hypothèse : dans celle de Warren au contraire, elle demeurait inexpliquée, ou plus, elle était un élément disqualifiant, dans la mesure où ces ossements impliquaient, et même s'ils n'étaient pas entaillés, une liaison quelconque avec les ossements d'enfants.

- soit ces fragments osseux avaient une fonction "religieuse», et leur enfouissement, voire leur éclatement et leur dispersion, pourraient répondre à une quelconque valeur magique, sacrée, protectrice voire propitiatoire : c'est cette hypothèse qui recueille ma préférence et c'est dans cette voie que j'invite les scientifiques à poursuivre leurs investigations.

16 Voir K. BRanigan, Ritual Interference with Human Bones in the Mesara Tholoi, in Thanatos (cité n. 12), p. 49-50: «some [bones] were certainly broken in small pieces and placed in a ritual context in the Early Bronze Age. In room I [du site de Ayia Kyriaki] a small area of undisturbed deposit was found and this contained twenty small fragments of bone, together with fragments of conical cups. This small deposit against the end wall of the otherwise empty room was far too small to represent any clearing deposit from the tomb chamber - that is, room I was not being used as dump for bones. The bones appear to have been selected and brought here for some specific piece of ritual». 
S'il se trouvait malgré tout des inconditionnels du cannibalisme cnossien au Minoen récent I B, il ne pourrait être question pour eux, pas plus que dans le cas d'Anemospilia, de clamer que l'anthropophagie rituelle était répandue dans l'île à cette époque ${ }^{17}$. Plus grave, et Hughes n'a que peu abordé la question, est l'interprétation que Warren propose de sa découverte en parallèle avec les sources littéraires des périodes classique et hellénistico-romaine; elle tient en peu de mots : qui dit pratiques cannibales, en effet, évoque la religion dionysiaque, que les textes associent volontiers au (dia-)sparagmos, démembrement d'une victime vivante, et à son corollaire, l'ômophagia ou ingestion des chairs crues. Les Bacchantes d'Euripide, éloquentes à souhait, sont invoquées au premier rang de nombreux témoignages rappelant de près ou de loin ces macabres pratiques, à Chios, à Ténédos, à Lesbos, à Potnia, ainsi que dans les légendes des Proétides, des Minyades et des Cadméides. Cependant, le culte dionysiaque est peu implanté en Crète, et la preuve en est que le nom du dieu n'est jamais repris dans les en-têtes des traités entre les différentes cités de l'époque classique. La raison de ce faible développement est qu'en Crète existait déjà un autre culte aux allures dionysiaques, celui de Zagreus, connu notamment d'Euripide, dans sa pièce intitulée Les Crétois. Cette divinité aurait été, dès l'Antiquité, identifiée au Zeus crétois et à Dionysos, tandis que son nom suggérerait une origine pré-grecque, minoenne. Un mythe racontait aussi comment, alors qu'il était enfant et gardé par les Courètes, - qui sacrifiaient des enfants à Cronos, - il avait été attiré et capturé par les Titans, pour être aussitôt démembré, mis à cuire et dévoré ${ }^{18}$.

Ce développement, rattaché déjà à une explication archéologique des plus douteuses, repose lui aussi sur des pieds d'argile : le fragment des Crétois n'est pas au-dessus de tout soupçon quant à la bonne information du Tragique ${ }^{19}$. Les légendes invoquées pour le sparagmos et

17 Comme le fait R. CASTLEDEN (The Knossos Labyrinth [cité n. 5], p. 122-123), quand il rapproche les faits archéologiques de la légende du Minotaure. Comme si quelques entailles inexpliquées sur des ossements étaient une base suffisante pour affirmer de telles manifestations cultuelles, et $a$ priori à grande échelle.

18 P.M. WARREN, Minoan Crete and Ecstatic Religion (cité n. 6), p. 161-163; bref commentaire de D. HuGHES, p. 254 et n. 24.

19 F. 472, Nauck 2 : on y trouve, rapidement associés, mystères de l'Ida et culte ômophagique en l'honneur de Zagreus. Le prêtre de Zeus parviendrait, selon ce passage, à concilier abstinence de toute nourriture jadis vivante et festins 
l'ômophagia, émanant pour la plupart d'auteurs post-classiques et chrétiens, ne prouvent déjà pas l'existence de ces pratiques dans le culte dionysiaque ${ }^{20}$, et même si une nouvelle fois on se hasardait à l'admettre, elles ne pourraient pas être transposées, sans autre forme de procès, dans le culte de l'éminemment obscur Zagreus ${ }^{21}$, lequel, s'il est démembré et dévoré, est également ramené à la vie et peut être ainsi le héros d'un mythe de renaissance de canevas classique. Enfin, il faut oser ce saut dans le vide que représente, en cette question tellement controversée de la continuité cultuelle durant les âges sombres, le lapsus chronologique d'un millénaire, sur des bases aussi faiblement assurées $^{22}$.

Si le cannibalisme est ainsi proposé comme la solution au mystère des incisions sur les pauvres vestiges humains de la North House, les faiblesses de cette hypothèse sont à la mesure de son caractère extraordinaire. Imaginons le sourire que nous réserverions à l'archéologue qui voudrait faire des reliquaires les œuvres d'art destinées à l'ostentation de la cruauté sacrificielle des chrétiens - qui mangent et boivent le corps et le sang du Christ - au cœur même de leurs lieux de culte : gardons-nous du risque d'énoncer pareilles aberrations à propos de réalités qui nous sont insuffisamment connues, tels les ossements incisés de Cnossos.

ômophagiques destinés à Zagreus : Euripide a visiblement uni plusieurs traditions crétoises distinctes, et en particulier celles de Zeus et de Zagreus.

20 Textes ou plutôt fragments de textes d'auteurs, dangereusement isolés de leur contexte, utilisés à des fins apologétiques par les chrétiens, ou par des paiens opposés au sacrifice sanglant : CLÉMENT D'AlEXANDRIE, Protreptique, III, 42; EUELPIS DE CARYSTOS apud PORPHYRE, De l'Abstinence, II, 55, 3. La tradition de sacrifice humain à Potnia n'a pas de réalité historique, ne comprend aucune trace de cannibalisme, et s'insère plutôt dans les mythes d'initiation à l'âge adulte, tandis que les histoires de Minyades, Proétides et Cadméides participent à la fois des mythes d'inversion dionysiaque et des mythes adolescents (voir ma thèse, à paraître).

21 Voir par exemple les réserves de M.L. West, The Orphic Poems (Oxford, 1983), p. 153-154, et celles de M.P. NILSSON, $M M R^{2}$, p. 578.

22 Lapsus dont Peter Warren connaît les dangers; il pense cependant pouvoir en déjouer les pièges en affirmant que la continuité religieuse peut exister dans les pratiques cultuelles, et qu'en ce cas précis son argumentation n'est pas si faible, étant donné que Zagreus, nom "pré-grec", renvoie au-delà des âges sombres, si pas directement à la période minoenne. Mais comment pourrait-on établir une liaison sûre entre ce Zagreus pré-grec et les "pratiques cultuelles» de Cnossos? 


\section{Des sacrifices humains lors des funérailles, en Grèce et à Chypre?}

Dennis Hughes se reporte ensuite sur les découvertes archéologiques de Grèce propre puis de Chypre, pour discuter des squelettes trouvés, dans les dromoi ou dans les chambres, en position inaccoutumée ou en des contextes jugés énigmatiques, et à propos desquels on a souvent eu recours, depuis la fin du siècle dernier, à l'hypothèse de l'immolation de serviteurs au profit de leurs défunts maîtres. Pour la facilité, je citerai, dans l'ordre adopté par l'auteur, les différentes tombes au sujet desquelles une précision pourrait s'avérer utile :

Mycènes, Ville basse, tombe 15 : la description de Tsountas laisse clairement entrevoir que les six cadavres enterrés dans le dromos, devant le triangle de décharge, l'étaient les uns sur les autres : pourquoi d'abord aurait-on d'abord rempli le dromos aux trois quarts et immolé des serviteurs à hauteur du linteau, plutôt que devant l'entrée ellemême? Cette situation se comprendrait mieux dans l'hypothèse d'une cérémonie postérieure où les fossoyeurs n'auraient pas pris la peine de creuser le remblai jusqu'au sol vierge. De plus, à supposer que les "esclaves" aient été rituellement exécutés, on aurait pu s'attendre à en trouver les corps sur un même niveau, et non tous superposés les uns sur les autres. Peut-être enfin s'agit-il simplement des anciens occupants de la chambre, grossièrement réinstallés dans le dromos après la réutilisation du caveau ${ }^{23}$.

Argos, La Deiras, tombe VI : Vollgraff s'exprime en ces termes ${ }^{24}$ : «(...) devant l'entrée du VIe tombeau, on a trouvé, à hauteur du sommet de la porte, des ossements humains ensevelis sous un monceau de pierres (...)». Mais le fouilleur n'a pris la peine de détailler ni le nombre, ni l'état des ossements, ni même leur disposition exacte : l'utilisation de son témoignage est donc ardue, et ouvre la porte à tous les abus.

Chypre, nécropole de Kastros, tombe 422 : Utilisée au cyprogéométrique I, ainsi que l'attestent un peu de poterie et quelques ossements épars recouverts d'une couche de terre, la chambre fut rouverte au cypro-géométrique III pour l'inhumation d'un homme, au côté duquel on avait déposé une pointe de fer. Le dromos reflète partiel-

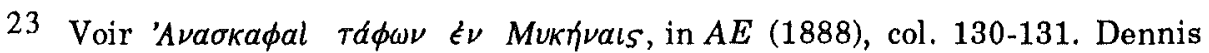
Hughes (Human Sacrifice, p. 31) énonce la même conclusion mais sans entrer dans le détail.

24 Fouilles d'Argos, in BCH, 28 (1904), p. 370 et n. 3. 
lement cette double utilisation : une couche de remblai conservait des tessons de poterie du cypro-géométrique I, une autre, homogène, correspondait au cypro-géométrique III, tandis qu'une troisième, de terre sablonneuse et noire, d'origine inexpliquée, recouvrait le tout. Afin de juger correctement les dires du fouilleur, laissons d'abord longuement la parole à Einar Gjerstad ${ }^{25}$ :

At the entrance of the dromos lay the remains of three skeletons placed one upon another, the uppermost covered from the breast to the feet by two stone slabs in the shape of a plank-shaped idol similar to those of the Cypriote Bronze Age, and a small slab of stone placed across his neck. When the stone slabs had been removed, it was found that the uppermost skeleton lay in an outstretched position with the head towards the South, but the shoulder-blades were on the top of the ribs, the processes of the spinal column were turned up, the hands were tied to each other and the feet were crossed. From this it was concluded that the body had been placed on its face with its hands and feet tied. Below the knees of the uppermost skeleton, the skull and scattered bones of the second were found. From the position of the bones it seems that the body has been placed there in a mutilated condition. Below this second skeleton, finaly, the remains of a third came to light; it was found in a damaged state, but it could be seen that the body had been placed in an outstretched position on its right side with the head towards the North. At the east side of the skeleton one jug and one amphora were found, having contained food and drink. The finding of these skeletons must be connected with that of a large rectangular block of porous stone, which was found lying obliquely in the uppermost dromos-filling above the door, with its upper edge reaching the right rock edge of the dromos. This block measures $1.18 \mathrm{~m}$. in length, $0.51 \mathrm{~m}$. in width, and $0.34 \mathrm{~m}$. in heigth. In the middle, a rectangular hole is cut, $0.23 \mathrm{~m}$. by $0.12 \mathrm{~m}$. From the short sides of this hole, hollow grooves run to the long sides of the block and down these to about the middle of them. At the place where the blocks ( $\mathrm{sic}$ ) were found, there were cuttings in the rock edge of the dromos, whereby it could be concluded that the block had originally been placed across the dromos. These facts seem to have to be explained in the following way: The stone block is a sacrificial table, where human sacrifices have been performed, the remains of which were the skeletons found. When the victims with their hands and feet tied had been killed, their blood poured down in the hole to satisfy the spirit of the deceased, buried in the tomb, and they were themselves buried at the entrance of the dromos (...). That the deceased, to whom these human sacrifices were performed, was the person buried in the second burial layer of the chamber, is evident both from the level of the skeletons and the sacrificial table, and the date of the two vases found in connection with the skeletons, as compared with the date of the finds of the first burial period.

25 Dans The Swedish Cyprus Expedition. Finds and Results of the Excavations in Cyprus, 1927-1931, 1, Stockholm, 1934, p. 244-245. 
Hughes, non sans évoquer ensuite certains doutes, conclut : «As the uppermost skeleton had clearly been bound, we have in tomb 422 good evidence of some sort of killing, and arguably a ritual killing» 26 .

L'hypothèse d'une immolation reste ici plausible, mais ne doit pas inversement être considérée comme certaine. Que le premier inhumé ait eu les poignets et les chevilles attachés n'est vraiment pas une certitude : les mains semblent avoir été posées traditionnellement devant le bassin, alors qu'on les aurait plutôt attendues derrière le dos; elles ne semblent pas assez rapprochées pour témoigner irrévocablement de la présence de liens; les pieds croisés peuvent résulter d'un ensevelissement rapide, ou de l'inhumation d'un corps gagné dans cette position par la raideur cadavérique, ou du transfert de ce squelette à partir d'une autre tombe; cette dernière solution pourrait expliquer également l'état très fragmentaire des deux autres squelettes, sans passer par la supposition fantaisiste de mutilations préalables à l'enterrement : que seraient devenues d'ailleurs les parties qui ont aujourd'hui disparu ? n'aurait-on pas dû plutôt les trouver dispersées à l'entour du tombeau ? La présence d'offrandes funéraires n'est pas non plus favorable à la thèse d'un sacrifice. Pourquoi encore les corps des immolés gisaient-ils loin des tables dites sacrificielles, lesquelles sont sans parallèle connu et en conséquence interprétées en fonction du seul contexte, et donc aussi de l'idée directrice qui guide le fouilleur dans son interprétation? Ces dernières enfin n'étaient pas in situ puisque le fouilleur précise qu'originally elles (ou une d'entre elles au moins) avaient été disposées en travers du dromos, tout en se gardant de préciser les circonstances lors desquelles ces «tables» auraient été déplacées ${ }^{27}$. Les idoles plates qui couvraient les ossements (à supposer qu'il s'en agit) ne sont pas uniques, et dans certaines tombes à chambres, il semble qu'elles aient fait office d'offrande ${ }^{28}$.

\section{Human Sacrifice, p. 38-39.}

27 Les tables dites sacrificielles sont très mal décrites par l'inventeur : les illustrations 90 et 92 et la figure 94.3 en laissent clairement apparaître deux, alors qu'elle seule est présentée dans le texte, et avec une confusion probable de l'auteur (étant donné les dimensions incompatibles entre la description, le plan et les photographies).

28 Exemple à Vouni-Bellapaìs : H. CAssimatis, Les rites funéraires à Chypre, in $R D A C$ (1973), p. 128. 
Einar Gjerstad n'est pas plus clair en $1980^{29}$, quand il parle d'une large pierre rectangulaire qui servit de table d'égorgement, et d'un autel où avaient été accomplis les sacrifices humains. Au milieu de la pierre (laquelle ?), un trou laissait passage au sang ruisselant. Quelle différence l'auteur établit-il entre un autel et une table d'égorgement? Gjerstad affirme encore que les trois "serviteurs" avaient eu les mains liées : comment peut-il l'affirmer sans laisser aucune place à l'ombre d'un doute, alors qu'il ne subsiste presque rien des deuxième et troisième squelettes?

Salamine de Chypre, tombe 2 : Selon Dennis Hughes, pour cette tombe, "some form of ritual killing seems indicated" 30 . Ce jugement, quoique nuancé, m'apparaît encore trop indulgent. Utilisée à la charnière du cypro-géométrique III et du cypro-archaïque I ancien, puis au cypro-archaïque récent, la tombe a été pillée et n'a donc pas livré d'indications importantes. Mais dans le dromos, pour la seconde période, Vassos Karageorghis a trouvé les squelettes de deux chevaux, ou mules, avec la structure d'un char, ainsi que les restes d'êtres humains :

Near the western side of the dromos and at a distance of about $2.50 \mathrm{~m}$. from the façade of the chamber there was an almost complete human skeleton buried in the filling of the dromos. It was lying flat, with the skull to the north and the feet to the south; the legs were close together and the hands were joined as if fastened together in front of the body. The skeleton was lying at a depth of about $60 \mathrm{~cm}$. from the surface of the cultivated soil, and ca. $45 \mathrm{~cm}$. from the surface of the filling of the dromos. The utmost care was taken to find out whether there was any pit or grave in which the skeleton was buried, but there was none; the soil all round it was exactly the same and of the same texture as that of the rest of the filling. At a distance of about two meters to the west of this skeleton there was a human skull and other skeletal remains, obviously dispersed by the plough. There was not a single object associated with these skeletons. Based on the stratigraphic evidence which we are able to obtain we may suggest that these skeletons were buried at the same time when the dromos was filled up with soil.

Après avoir, en toute correction, dressé ce constat, l'auteur tente, dans la seconde partie de son livre, d'en dégager le sens :

If our interpretation of the human burials in the dromos of Tomb 2 is correct, then we have for the first time at Salamis an example of a slave burial. This, however, is not the first example in Cyprus; slave burials have been known

29 Ages and Days in Cyprus, Göteborg, 1980 (SIMA, 12), p. 44-45.

30 Human Sacrifice, p. 40. 
since the Late Bronze Age. A human skeleton was found in the dromos of a Late Cypriote II tomb at Enkomi, and this has been interpreted, though cautiously, as wa servant's tomb". Slave burials are also recorded from an early Iron Age tomb at Lapithos (beginning of the first millennium). It has been suggested that this is a custom which was introduced to Cyprus by the Greek colonists at the end of the Bronze Age, and it may have survived down to the Archaic and Classical periods, though it was not frequently practised. There is interesting literary evidence for human sacrifices in Cyprus, and particulary about Salamis. Slave burials were known in Mycenaean Greece, and there is an allusion to this custom in the Iliad; they are also known from Skythia, in association with horse burials 31 .

J'ose à peine mettre en doute les observations de cet extraordinaire archéologue qu'est Vassos Karageorghis, mais deux choses me semblent dignes d'attention. La carcasse des chevaux se trouvait in situ, à deux ou deux mètres cinquante de profondeur, tandis que les restes humains n'étaient enterrés qu'à une cinquantaine de centimètres; dans le cas d'un sacrifice conjoint et de surcroît rapproché des funérailles homériques, il faudrait expliquer pourquoi les hommes n'avaient pas été immolés en mêmes temps et lieu que les destriers ${ }^{32}$. D'autre part, si le dromos n'a pas été rouvert, qu'est donc devenu le reste des squelettes incomplets ? A moins de recourir encore à cette supposition très insatisfaisante d'une mutilation préalable, ces ossements isolés ne semblent pouvoir provenir que d'inhumations antérieures ou postérieures. La position des mains sur le pelvis enfin n'est certes pas inhabituelle lors des inhumations, et n'implique aucunement que les poignets fussent liés ${ }^{33}$.

La stratigraphie n'était d'ailleurs peut-être pas aussi nette que présentée : le fouilleur dit explicitement que les squelettes incomplets ont été dispersés par les labours; si l'on suppose que des enterrements secondaires ont été réalisés très rapidement après l'obturation du dromos, avant le tassement de la terre du remblai, les très faibles

31 Excavations at the Necropolis of Salamis , 1, Nicosie, 1967 (Salamis, 3), p. 8-11 [un squelette complet et "other skeletal remains"] et 121 . Voir aussi ID, Chronique des fouilles à Chypre en 1962, in BCH, 87 (1963), p. 373-380 [un squelette complet et les vestiges de deux autres]; ID., Ten Years of Archaealogy in Cyprus, 1953-1962, in $A A$ (1963), col. 549-550.

32 Élément répertorié comme étrange par HuGHEs, Human Sacrifice, p. 40.

33 La position des mains sur le bassin est une des constantes dans les inhumations humaines, et les exemples abondent, en Grèce comme partout dans le monde, des temps préhistoriques à nos jours. Je ne vois pas comment on pourrait valablement faire de cet élément l'indice d'une cérémonie de sacrifice humain. 
différences de couches ont pu être réduites à néant par les travaux agricoles postérieurs ${ }^{34}$. Les séquences et les modalités d'utilisation de la chambre demeurent obscures, et la tombe royale 2 de Salamine, si elle peut être considérée comme ayant livré un des rares cas archéologiques acceptables de sacrifice humain, n'offre certainement pas la preuve attendue en la matière.

De plus, l'interprétation de V. Karageorghis apparait assez fortement dépendante de l'historiographie antérieure, problème d'importance capitale sur lequel nous aurons à revenir.

Chypre, Tamassos, tombe 11 [4] : les fouilles de Tamassos, réalisées au XIXe siècle par Ohnefalsh-Richter, ne sont connues que par un bref article du $B C H$, dû à Olivier Masson : les documents originaux de ces fouilles, longtemps considérés comme détruits, sont en cours de publication. La tombe 11 est habituellement datée de la fin du cypro-archaiqque I, et son dromos aurait livré des os chevalins et humains. Après avoir rassemblé les notes du fouilleur, l'auteur ajoute ${ }^{35}$ :

Selon toute probabilité, il s'est agi à Tamassos, comme dans la tombe 2 de

Salamine, d'esclaves sacrifiés au moment de l'inhumation.

L'ensemble toutefois, même s'il est présenté avec beaucoup d'autorité, est trop peu connu pour être interprété définitivement, ainsi que le souligne Hughes ${ }^{36}$ : on ne dispose d'aucune donnée stratigraphique, ni même d'indications précises sur la proximité éventuelle des ossements animaux et humains.

De plus, Masson, qui cite Karageorghis pour conforter son rapprochement, le cite erronément : les squelettes de la tombe 2 de Salamine ne sont pas «assez profondément enfouis», ce qui les associerait directe-

34 Une critique de ce genre avait déjà été formulée en 1963 par P. DikaIos, $A$ "Royal Tomb" at Salamis, Cyprus, in AA (1963), col. 126-210, au vu d'une comparaison avec le dromos de la tombe I de Salamine, creusé de nombreuses fosses postérieures à l'utilisation de la chambre. V. KARAGEORGHIS (Nouvelles découvertes dans la nécropole royale de Salamine de Chypre, in Le rayonnement des civilisations grecque et romaine sur les cultures périphériques, Paris, 1965, p. 311, note additionnelle; ID., Cyprus. From the Stone Age to the Romans, Londres, 1982, p. 135) a rejeté sans appel cette possibilité, les couches archéologiques n'ayant subi, selon lui, aucune modification.

35 Kypriaka. I. Recherches sur les antiquités de Tamassos, in BCH, 88 (1964), p. 220-221.

36 Human Sacrifice, p. 40. 
ment avec les os des chevaux, mais «assez peu profondément enfouis», ce qui les en distingue nettement ${ }^{37}$. Aussi, le rapprochement en est-il passablement affaibli et les conclusions avancées à propos de la tombe de Tamassos considérablement diminuées.

\section{Divers}

Hughes enfin a laissé échapper quelques témoignages intéressants, ou en a tiré un parti parfois un peu décevant.

Ainsi l'effervescence suscitée par les trouvailles de Cnossos incita les archéologues à reporter leur attention sur quelques fragments de squelettes exhumés dans certaines fouilles minoennes, et dont la signification restait imprécisée. À Myrtos, établissement du minoen ancien II, l'équipe de Peter Warren avait trouvé, à la fin des années soixante, de quoi reconstituer le quart de la calotte crânienne d'un jeune individu. Les fragments d'os, ainsi que la céramique avoisinante, avaient subi les effets du feu lors de l'incendie qui détruisit le site ${ }^{38}$ :

Thus the possibilities of ancestor worship (cf. the plastered skulls of Neolithic Jericho) or even human sacrifice cannot be ruled out (...). The skull would have had some functions in the rites.

Dans un contexte analogue, Nicolas Platon avait découvert un crâne d'enfant près du "sanctuaire central" du palais de Zacros et évoqué de même la possibilité d'une immolation humaine ${ }^{39}$. Dans une chambre de la maison I de Trianda, à Rhodes, établissement traditionnellement reconnu pour minoen, reposaient éparpillés, dans les coins est et nordouest, des crânes, des mâchoires, des phalanges de la main (?), contemporains des ossements de Cnossos 40 .

La thèse du sacrifice humain n'offre pas davantage, en ces cas précis, la solution idéale : d'abord, elle doit être inférée, - et le pas est grand à franchir -, à partir de la seule existence d'un ou de quelques fragments d'os. Ensuite, on pourrait s'attendre, si immolations il y avait eu, à retrouver des squelettes, si pas entiers, du moins un peu plus complets. Troisièmement, ces interprétations souffrent de comparai-

37 Kypriaka (cité n. 35), p. 220.

38 P.M. Warren, Myrtos. An Early Bronze Age Settlement in Crete, Londres, 1972 (ABSA Suppl. 7), p. 82-83.

39 N. Platon, Zakros. To neon minôt'kon anaktoron, Athènes, 1974, p. 105.

40 Cas cité par S. HEUCK, in Sanctuaries and Cults (cité n. 6), p. 212. 
sons insuffisantes : les ossements trouvés dispersés et sans raison logique ne sont pas rares à l'âge du Bronze. Enfin l'hypothèse d'une vénération cultuelle, qu'elle soit d'un ancêtre ${ }^{41}$, ou plutôt d'enfants morts selon certaines circonstances, - et dont la possession des ossements aurait été bénéfique, ou encore aurait nécessité mesures expiatoires ou propitiatoires - fait partie des conjectures tout aussi autorisées. Enfin et surtout, si pour chaque découverte d'un os dont la présence est difficilement explicable il fallait se résoudre à l'invocation d'immolations humaines, l'Histoire connue à partir des seuls témoignages archéologiques se résumerait à n'être qu'une vaste histoire de sacrifices humains 42 .

Asinè. Les tombes conjointes 80 et 81 de l'importante nécropole d'Asinè, fouillée de 1922 à 1930 par Axel Persson et Otto Frödin, confrontèrent les fouilleurs à une curieuse disposition funéraire. Le squelette d'une femme recroquevillée dans la mort reposait au fond de la ciste 80 , mais les dalles de couverture de la fosse formaient au nord-est un saillant d'une cinquantaine de centimètres, lequel abritait le squelette incomplet d'une autre femme, qu'une note infrapaginale, citée in extenso, définit en ces termes:

Probably the remains of a slave-woman sacrified to the dead.

41 L'hypothèse du culte rendu à un ancêtre, évoquée par Warren pour le cas de Myrtos, ne semble pas probante, elle non plus, en ce sens que le crâne trouvé à Myrtos était celui d'un être jeune. Sans doute est-il plus opportun, ici comme à Cnossos et à Zacros, d'assigner aux ossements une fonction religieuse, encore énigmatique.

42 Dennis Hughes (Human Sacrifice, 196-198) a catalogué une série de cas à part, sous la rubrique Some Unexplained Mass Burials. On y trouve plusieurs exemples d'inhumation collective qui auraient pu, si leurs inventeurs avaient eu tendance à recourir sans hésitation au thème du sacrifice humain, être interprétés en ce sens. Un puits corinthien de l'helladique ancien III renfermait ainsi, en même temps que des ossements d'animaux en tous genres, plus de vingt squelettes d'hommes, adolescents, adultes et vieillards. A Nichoria, en Messénie, une fosse commune de l'helladique récent II A contenait onze squelettes, hommes, femmes et enfants, enterrés sans aucun soin ni offrande funéraire. Un puits argien de l'helladique récent III C a livré, en 1971, vingt squelettes, mêlés comme à Corinthe avec des ossements animaux. Il est vraisemblable que bien d'autres cas de ce genre sommeillent encore au fond des rapports de fouilles. Ainsi par exemple le cas des suppliciés de Fourni, à Délos, dont les squelettes ont été retrouvés en pleine ville (P. et N. DUCREY, Les suppliciés de Fourni, in Etudes déliennes, Paris, 1973 [BCH Suppl. 1], p. 173181). 
Si, de l'aveu même des auteurs,

unfortunately no close investigation as to the position of the skeleton was made, or could be made, or into its relation to Tomb M.H. 80,

rien ne les autorisait à réaffirmer, et dans le corps du texte cette fois :

a great deal speaks in favour of its being the remains of a slave-woman sacrified to the woman buried in the last-mentioned tomb [c'est-à-dire la tombe $80]^{43}$.

L'interprétation ne se fondait donc sur aucun élément de preuve. On a dit que le squelette de la tombe 81 était incomplet : peut-être les inhumants ont-ils trouvé, à l'emplacement de la future tombe 80 qu'ils étaient en train de creuser, des os encore articulés provenant d'une inhumation précédente, et qu'ils auraient traités avec respect, en les recouvrant, sans aucun doute expressément, par les plaques de pierre grâce auxquelles ils se préparaient à fermer aussi la tombe voisine.

Vrana. L'agencement des quatre tumuli de Vrana, près de Marathon, fouillés en 1970 par Spyridon Marinatos, a été réanalysé par Olivier Pelon, et en particulier celui du quatrième cercle funéraire. D'un diamètre de 16,85 mètres, il renferme en sa partie nord-est un cercle plus petit, non tangent, contenant deux fosses, dont la tombe principale du site. Dans le grand cercle reposaient six tombes anciennes, dont deux en position rayonnante vis-à-vis du petit cercle. La première contenait les os d'une femme au statut élevé, nommée reine, et la seconde la cage thoracique et la tête d'un cheval ${ }^{44}$ :

La disposition des tombes du tumulus I paraît (...) conduire à l'hypothèse d'une sépulture de chef avec sacrifice accompagnateur, animal et peut-être humain.

La simultanéité des inhumations centrales et périphériques ne saurait être établie et en l'absence d'autres éléments, pareille supposition reste sujette à caution.

Athènes. Pour l'époque historique, une nécropole, située entre l'Aréopage et la colline des Nymphes, a livré les restes de dix-sept bûchers funéraires. Ceux-ci contenaient de petits vases et des débris

43 O. Frödn et A.W. Persson, Asine. Results of the Swedish Excavations, 19221930, Stockholm, 1938, p. 124 (et n. 3)-125.

44 O. PELON, Tholoi, tumuli et cercles funeraires. Recherches sur les monuments funéraires de plan circulaire dans l'Égée de l'âge du bronze (IIIe-IIe millénaires av. J.-C.), Paris, 1976 (BEFAR, 229), p. 83-84 et 106. 
osseux, animaux et humains ou, plus exactement, d'enfants en très bas âge, ossements dont la faible ossification explique la très mauvaise conservation ${ }^{45}$. Ils datent des IVe et IIIe siècles avant J.-C., et leur présence à l'intérieur de la cité, qui interdisait toute inhumation en ses murs ${ }^{46}$, ne laisse pas de paraitre mystérieuse, surtout que ceux-ci présentent certaines analogies avec les tophets puniques. Rodney S. Young avait supposé que l'interdiction d'inhumer à l'intérieur des murs ne valait pas pour les enfants, mais puisqu'inversement de nombreuses nécropoles contiennent également des vestiges enfantins, cette supposition perd beaucoup de sa valeur. D'ailleurs, la coutume voudrait plutôt que les enfants soient enterrés, et non incinérés comme les adultes ${ }^{47}$. D'où, deux solutions, fondées chacune sur cette idée que les enfants inhumés en ville appartiennent à une catégorie particulière qui échappait à ladite interdiction : ces nouveau-nés seraient soit des enfants morts prématurément, qu'on avait pas eu le temps d'intégrer aux cadres familiaux, soit des enfants volontairement rejetés par exposition dès la naissance, autrement dit, de toute façon, des enfants exclus et non reconnus au statut d'êtres humains ${ }^{48}$. La combustion des petits corps aurait été idéale d'un point de vue hygiénique et pourvue d'une valeur cathartique, laquelle serait présente également dans les sacrifices animaux et l'offrande des petits vases trouvés sur place ${ }^{49}$.

Nauplie. Enfin, un squelette d'enfant a été trouvé dans une fortification de Nauplie, sous le seuil d'une porte, datée du IIIe siècle après J.-C. Le sacrifice de fondation a été cité, il y a peu, comme l'explication la plus adéquate de cette trouvaille, étrange essentiellement par sa date tardive :

Here a child had been sacrified and placed underneath a threshold in one of the towers. Such measure to ensure strength and durability were surely more widespread than the archaeological record suggests.

45 R.S. Young, Sepulturae intra urbem, in Hesperia, 20 (1951), p. 67-134. La taille des bûchers est très petite ( $80 \times 115$ centimètres pour le plus grand).

46 CiCÉRoN, Lettres, 4, 12, 3. Interdiction vérifiée dans les faits par les fouilles américaines : voir R.S. YouNG, Sepulturae intra urbem (cité n. 45), p. 67-134.

47 J. RUDHARDT, Sur quelques bûchers d'enfants découverts dans la ville d'Athènes, in $M H, 20$ (1963), p. 15-16.

48 J. RUDHARDT, Sur quelques bûchers d'enfants (cité n. 47), p. 16-20. Voir aussi D.C. KuRTZ et J. BOARDMAN, Greek Burial Customs, Londres, 1971 (Aspects of Greek and Roman Life), p. 74 et 99.

49 J. RUDHARDT, Sur quelques bûchers d'enfants (cité n. 47), p. 19-20. 
Le parallèle des immolations similaires en Orient ancien est sousjacent, et l'affirmation que cette pratique était certainement plus répandue que les découvertes archéologiques ne le laissent présumer, a été clamée haut et fort ${ }^{50}$.

Je doute fortement qu'il existe, dans ce Moyen-Orient si souvent cité en référence, aucune preuve de ce que les enfants, dont les squelettes ont été trouvés dans les fondations, sous les cours intérieures et même sous les pavements, aient été effectivement immolés. Ce qui ne souffre aucun doute, par contre, c'est que cette façon de voir était la seule que connaissait l'historiographie du début du siècle, pour ces régions comme pour la Grèce, nous aurons à y revenir ${ }^{51}$. En Argolide, dans la maison D de la ville basse d'Asinè, des corps d'enfants ont également été disposés à ces endroits, lors de l'helladique moyen, mais l'inventeur, G.C. Nordquist, incline avec sagesse en faveur d'autres significations religieuses ${ }^{52}$.

Que le squelette, ou l'esprit qu'il représente ou auquel il renvoie, ait effectivement rempli une fonction favorable à la longévité ou à la solidité de l'édifice paraît probable, mais comment affirmer qu'il y ait eu sacrifice ? Il pourrait plutôt s'agir de l'ensevelissement d'un enfant décédé et dont la possession de la dépouille aurait été bénéfique, pour une raison qui en partie nous échappe, comme j'ai dit que cela pourrait être le cas des ossements entaillés de Cnossos.

50 B. Wells, Early Greek Building Sacrifices, in Early Greek Cult Practice. Proceedings of the Fifth International Symposium at the Swedish Institute at Athens, 26-29 June, 1986, Stockholm, 1988 (ActaAth, 38), p. 264-265.

51 Voir par exemple L.H. VINCENT, Canaan d'après l'exploration récente, Paris, 1907 (Etudes bibliques), p. 50-51; 196-201. L'ouvrage de A.R.W. GREEN (The Role of Human Sacrifice in the Ancient Near East, Missoula, 1975 [American School of Oriental Research, Diss. Ser., 1]) reste encore très fortement influencé par ces conceptions du début du siècle, et n'apporte, à mes yeux, aucune preuve à l'existence du sacrifice humain au Moyen-Orient.

52 A Middle Helladic Village. Asine in the Argolid, Uppsala, 1987 (Boreas, 16), p. 95. 


\section{Le rôle de l'historiographie de la fin du XIXe et du début du XXe siècles sur le concept de "sacrifice humain archéologiquement attesté"}

Hughes évoque succinctement l'influence qu'eut sur les archéologues le jugement de Tsountas à propos des sacrifices humains dans les tombes mycéniennes ${ }^{53}$. Je pense qu'il faut aller plus loin, et proposer une remise en question de la méthode en vertu de laquelle toute l'historiographie moderne a accepté, avec une homogénéité remarquable, l'existence de quelques rares sacrifices humains chez les Mycéniens et les Cypriotes.

Figure 2: Filiation historiographique en matière de sacrifices humains grecs prétendûment attestés par l'archéologie. Les flèches indiquent la dépendance des auteurs vis-à-vis de publications plus anciennes.

(voir page en regard) 


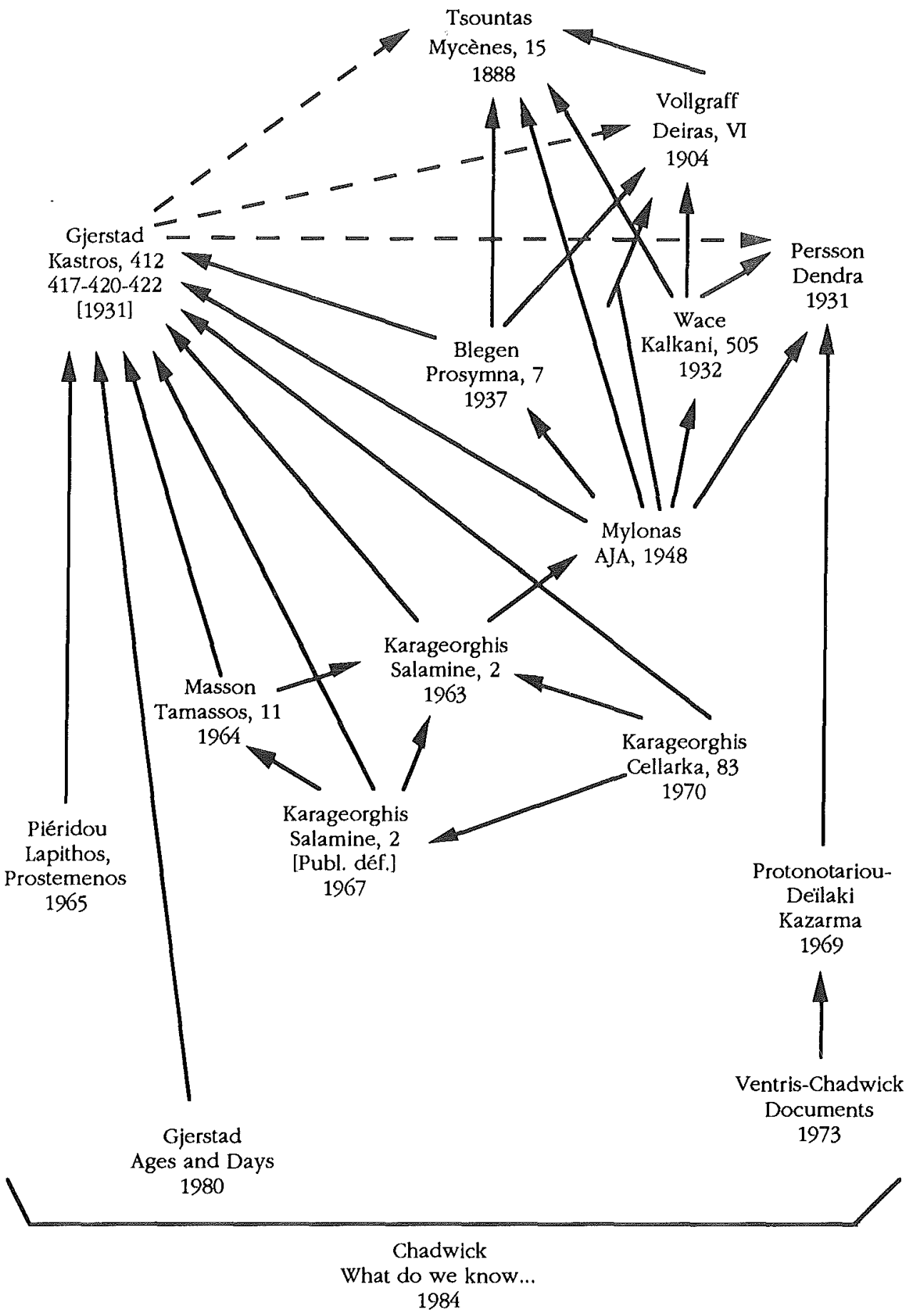


En ce qui concerne les découvertes d'avant-guerre, Carl Blegen 54 fut le dernier, en 1937, à citer un cas d'immolation dans sa publication des fouilles de la nécropole de Prosymna. Dans le doute, celui-ci avait assuré son affirmation en se référant aux cas de sacrifice humain dont il avait connaissance, à savoir celui de Mycènes (1888, Tsountas), celui de la Deiras (1904, Vollgraff) et ceux des dromoi des tombes de Chypre (1931, Gjerstad); Alan Wace, cinq années auparavant ${ }^{55}$, pour étayer un très hypothétique cas de sacrifice humain dans ses fouilles de Kalkani, s'était armé de l'autorité de Persson (1931, Dendra), de Vollgraff et de Tsountas. Einar Gjerstad, membre de l'expédition suédoise à Chypre de 1927 à 1931, concluait sans détours, sans preuves et à partir d'indices dérisoires, à l'origine mycénienne des sacrifices humains dans les tombes de Chypre : il ne cite aucune référence, mais puisqu'il croit aux sacrifices humains d'Argolide, il devait se référer à Persson, Vollgraff et Tsountas, seuls auteurs à avoir alors parlé d'immolations humaines dans les usages funéraires mycéniens ${ }^{56}$. Wilhelm Vollgraff, bien qu'en présence de fort peu d'éléments, s'était reporté, en 1904, à l'exemple alors unique de la tombe 15 de Mycènes. Et comme si ce dernier avait valeur d'exemple définitivement démontré, tous les auteurs, sans exception hésitants quant à leur trouvaille, y remontent avec unanimité, en y ajoutant les découvertes postérieures, elles aussi devenues intouchables car suspendues par rapprochement à l'autorité du savant grec.

54 Prosymna. The Helladic Settlement Preceding the Argive Heraion, 1, Cambridge, 1937, p. 156-157; 235-236.

55 Chamber Tombs at Mycenae, Oxford, 1932 (Archaeologia, 82), p. 145-146.

56 The Swedish Cyprus Expedition (cité n. 25), 4 (2), Stockholm, 1948, p. 433. Gjerstad n'avait pas changé d'avis en 1980 : voir Ages and Days (cité n. 29), p. 44. Aux nombreux auteurs qui ont accordé crédit à la thèse d'une importation mycénienne du sacrifice humain funéraire à Chypre, cités par HUGHES (Human Sacrifice, p. 214-215, n. 102), on ajoutera P. ÅsTRöM, L'art et la culture antique de Chypre, in J. ThimmE, P. Åström, G. LILliU et J. WIESNER, Civilisations anciennes du bassin méditerranéen. II. Les CycladesChypre-Malte-La Syrie ancienne, trad. R. Latour, Paris, 1971 (L'art dans le Monde), p. 74; V. KARAGEORGHIs, Chronique des fouilles à Chypre en 1962 (cité n. 31), p. 378; ID., Excavations at the Necropolis of Salamis (cité n. 31), p. 121; E. SJÖQ̨UIST, Problems of the Late Cypriote Bronze Age, Stockholm, 1940, p. 27. 
Or ce dernier, rappelons-le, avait lui-même condamné, en 1928, son interprétation de départ, mais, au dire de Mylonas, en privé ${ }^{57}$. De même Persson, derrière l'autorité duquel Wace s'était abrité, allait radicalement revoir ses positions en $1938^{58}$; quant aux découvertes suédoises, elles ne valent qu'en fonction de l'interprétation que leur conférèrent les fouilleurs, et le sacrifice humain à Chypre, dans les dromoi, est tout sauf un élément certain : rien ne permet en tout cas pareille conclusion au vu des résultats des fouilles; un corpus de cas, où en apparence chaque exemple semblait assurer les autres, s'était cependant forgé, et toutes les interprétations, bien que ne reposant sur aucun élément solide, furent avec le temps considérées comme des quasi certitudes.

C'est à ce corpus que se référeront à leur tour Olivier Masson (Tamassos), Vassos Karageorghis (Salamine, tombe 2; Cellarka, tombe 83), puis Evangelia Protonotariou-Deïlaki (Kazarma). Karageorghis, en 1970, alors qu'il proposait de voir dans le cimetière de la Cellarka un cas de sacrifice humain ${ }^{59}$ en appelait à l'exemple de la tombe 2 de Salamine et aux conclusions de l'expédition suédoise; à propos de sa découverte à Salamine, en 1967 et déjà en $1963^{60}$, il produisait les références de la tombe 422 de Kastros, de celle de Tamassos et les travaux cités dans un article de Georges Mylonas ${ }^{61}$, à savoir de Tsountas, Vollgraff, Persson, Wace, Blegen et l'expédition suédoise à Chypre. Pour justifier ses interprétations au sujet de la tombe 4 de Tamassos, Masson proposait comme exemples décisifs les tombes

57 Voir G.E. Mylonas, Ancient Mycenae. The Capital City of Agamemnon, Princeton, 1957, p. 83; ID., Homeric and Mycenaean Burial Customs, in AJA, 52 (1948), p. 72; ID., Mycenae and the Mycenaean Age, Princeton, 1966, p. 116117.

58 Asine (cité n. 43), p. 356. On pressent combien le recours à l'hypothèse du sacrifice humain, pour certains ossements dont l'interprétation apparaissait incertaine, était fortement ancré quand on voit le même Axel Persson, dans ce même ouvrage où il récuse l'hypothèse de la mise à mort de "portiers" préposés à la quiétude des morts, en apppeler encore au sacrifice d'une esclave servile... (voir supra, p. 43).

59 Excavations at the Necropolis of Salamis , 2, Nicosie, 1970 (Salamis, 4), p. 123$126 ; 208 ; 232$.

60 Excavations at the Necropolis of Salamis, 1 (cité n. 31), p. 121 et Chronique des fouilles à Chypre en 1962 (cité n. 31), p. 378.

61 Homeric and Mycenaean Burial Customs (cité n. 57), p. 72. 
royales 1 et 2 de Salamine, ainsi que la tombe 422 de Kastros ${ }^{62}$. Madame Protonotariou-Deïlaki citait le cas de Dendra et évoquait le cercle B de Mycènes (allusion que pas plus que Hughes je n'ai réussi à élucider). Michael Ventris et John Chadwick se référaient à la découverte d'Evangelia Protonotariou-Deilaki63. En 1984, Chadwick faisait simplement référence aux découvertes dans les dromoi, sans précision aucune et comme si le fait était désormais prouvé ${ }^{64}$.

C'est que la vérité proposée par les rapports de fouilles, préliminaires ou définitifs, est armée d'un double tranchant : les découvertes peuvent avoir été consignées objectivement, mais souvent leur consignation n'a vu le jour qu'incorporée aussitôt dans une interprétation dont l'auteur se dispense volontiers de justifier le bien-fondé : à la critique épineuse des sources s'adjoint ainsi une difficile critique du témoignage unique de l'inventeur, et le dévoilement de la vérité historique en pâtit inévitablement.

Trois exemples de consignation interprétative valent la peine d'être évoqués, parce qu'ils sont puisés dans les œuvres d'archéologues dont la qualité est très justement reconnue :

Le premier est de la plume de Gjerstad, à propos de la tombe 422 de Lapithos qui, je le rappelle, est considérée encore aujourd'hui comme un des cas les moins discutables en matière de sacrifice humain attesté par le biais de l'archéologie. L'auteur s'étonne de la pauvreté des offrandes déposées dans le caveau; les trois corps enterrés dans le dromos lui permettent de trouver sur le champ une solution qui convient à son interprétation intuitive :

62 Kypriaka (cité n. 35), p. 221. Le dromos de la tombe 1 de Tamassos, fouillée par P. Dikaios, avait été creusé de plusieurs puits en vue d'y accomplir des enterrements secondaires. Alléguant que les couches du dromos avaient été fortement dérangées, Masson critique l'interprétation de l'inventeur, et suppose qu'à la tombe 1 comme à la tombe 2 des êtres humains avaient été sacrifiés lors des funérailles : il est à la fois surprenant et révélateur, alors que les hésitations stratigraphiques rendent tout jugement aléatoire, que Masson ait délibérément opté en faveur de l'hypothèse d'un sacrifice humain.

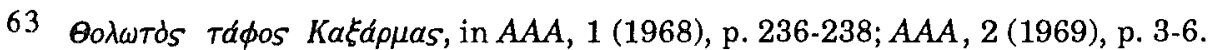
M. Ven'tris - J. ChaDWICK, Documents in Mycenaean Greek, Cambridge, $1973^{2}$, p. 460.

64 What do we know about Mycenaean Religion?, in Linear B : A 1984 Survey, éds A.M. DAvies et Y. DuHoux, Louvain-la-Neuve, 1985, p. 200. 
no tomb-gift in pots had been offered to the deceased warrior [qui reposait dans la chambre]. In compensation sacrifice of human victims were performed and remains of these were found in the dromos (... 65 .

En conséquence de quoi une certaine méfiance à l'égard des affirmations catégoriques de sacrifices humains dans les dromoi cypriotes apparaîtra du meilleur aloi. Je suppose que si de nombreuses offrandes avaient été découvertes dans la chambre, Gjerstad en aurait conclu aux grandioses funérailles d'un seigneur local, avec immolation de serviteurs...

Mais, plus encore, les exemples procurés par les deux grandes découvertes de l'été 1979 s'avèrent instructifs : Yannis Sakellarakis, dans son article du National Geographic, relate pas à pas sa découverte, et se hasarde à signaler qu'au moment même de la fouille, c'est-à-dire à un moment où il ne connaît pas l'ensemble des données ni les résultats de l'analyse complète de chaque pièce archéologique, il déclara : «It is hard to believe, but I think we have found a human sacrifice" : l'hypothèse du sacrifice humain était donc née avant même la consignation des faits, au cœur même de la découverte sur le terrain.

L'empressement malheureux des Sakellarakis se retrouve également dans le chef de Peter Warren, puisque celui-ci présenta, en 1979, dès après la première campagne de fouilles et de même sans être en possession de tous les éléments du "puzzle» de la North House, une communication à l'Institut suédois d'Athènes, intitulée déjà Minoan Crete and Ecstatic Religion : c'était sans doute prématuré, quand on sait que le rapport définitif ne parut dans l'Annual de l'École anglaise qu'en 1986. De plus, certaines des interprétations de Peter Warren semblent l'avoir conduit à une explication difficilement compréhensible des éléments que lui fournissaient ses fouilles : la figure 1 (voir supra, p. 31), qui montre l'éparpillement des fragments d'ossements crâniens dans les couches inférieures du niveau qui livra les vestiges humains, au premier coup d'œil, me semble interdire l'idée que des corps entiers avaient été déposés dans la pièce; Warren l'avait compris dans sa première publication ${ }^{66}$, quand il déclarait que des ossements épars avaient été jetés dans la chambre. Mais dans sa théorie à propos du cannibalisme, l'idée plus conforme à cette dernière que des corps

65 The Swedish Cyprus Expedition, 4 (2) (cité n. 56), p. 244 (les italiques ont été rajoutés par mes soins).

66 Apud H.W. Catling, Archaeology in Greece, 1979-80 (cité n. 1), p. 49. 
entiers avaient été abandonnés l'emporta ${ }^{67}$, pour être éliminée dans la publication définitive de $1986^{68}$, mais rester un des éléments majeurs et non explicitement réfuté, lui et ses conséquences, de l'explication anthropophagique qui n'a pas fait, dans cette publication finale, l'objet de correctifs : s'ajoute en plus, ici, la dualité de sens conférée par une même personne à un même indice archéologique.

En des matières aussi brûlantes, la prudence doit rester de mise, car toute mauvaise interprétation prête à conséquence, en ce qu'elle influence nombre d'auteurs dont la confiance est abusée : le dommage sera d'autant plus grave que le sacrifice humain est un sujet qui touche notre sensibilité et notre émotivité.

Dennis Hughes a souvent souligné que pour conclure au sacrifice humain dans le culte des morts, la simultanéité de l'ensevelissement du "maître" dans la chambre et celle, dans le dromos, des prétendus immolés, doit avoir été prouvée : cette vérité, digne de Monsieur de La Palice mais si fréquemment bafouée, est essentielle pour toute hypothèse faisant appel au sacrifice humain. Sinon, comme pour la tombe 11 d'Enkomi, on prend le risque de parler de cérémonies sacrificielles alors que les inhumations pourraient y avoir été respectivement accomplies à des époques différentes, parfois fort éloignées dans le temps ${ }^{69}$. Soit dit en passant, la constatation du dégagement intégral des remblais du dromos ne pourrait davantage, à elle seule, apporter la preuve de la simultanéité des inhumations au-dedans et au-dehors de la chambre : la voie d'accès peut également avoir été évidée aux fins d'un nouvel ensevelissement en dehors de la pièce funéraire, ou encore d'un enterrement d'abord destiné à prendre place dans le caveau mais effectué par la suite à l'extérieur.

À ces cas d'ensevelissements dans les dromoi contemporains de l'abandon des tombes, ou postérieurs à celui-ci, viennent s'adjoindre les cas où les ossements des premiers occupants des chambres ont été

67 Knossos : Stratigraphical Museum Excavations (cité n. 6), p. 91.

68 S.M. Wall, J.H. Musgrave et P.M. WarRen, Human Bones from a Late Minoan I B House (cité n. 6), p. 341-342.

69 The Swedish Cyprus Expedition, 1 (cité n. 25), p. 515, puis 524-525 et 575. 
transportés, avec ou sans respect, de celles-ci dans la descente : des quantités notables d'ossements ont ainsi été retrouvées dans les dromoi, qui proviennent d'inhumations antérieures. Certains squelettes, ou certaines parties de squelettes, ont pu être transportés d'un seul tenant au dehors, parce qu'encore transportables. Ainsi pourrait s'expliquer la présence de squelettes humains incomplets, pour lesquels on a parfois eu recours, sans grande certitude d'ailleurs, à cette supposition d'une mutilation préalable.

$\mathrm{Au}$ reste, lorsqu'on jette un rapide coup d'œil sur l'ensemble des sources, rien ne vient conforter l'idée d'une «tradition sacrificielle», fixée dans ses grandes lignes par la coutume : c'est que les ressemblances n'abondent pas pour fournir aux cas étudiés une unité, même apparente. Hughes le signale, mais il n'est pas vain de reprendre chaque cas en détail : ainsi, les victimes auraient parfois été abattues dans la chambre et reposé à même le sol, comme à Kazarma, à Vouni et à Dendra, où un autre sacrifié aurait lui bénéficié d'une fosse; parfois elles auraient été tuées juste au-devant de la porte, au niveau du sol, comme à Kalkani, à la Cellarka de Salamine, parfois dans l'entrée même de la chambre, comme à la tombe 417 et au Prostemenos de Lapithos; voire encore devant le linteau, à plusieurs mètres du niveau de la pente, comme à la tombe 15 de Mycènes, à la Deiras et à Prosymna; d'autres auraient été enterrées en plein milieu du dromos, ainsi qu'à la tombe 420 de Kastros, à la tombe 2 de Salamine, à Tamassos (?) et à Enkomi. Enfin, les "prisonniers» de la tombe 422 de Kastros, abattus cruellement au bas de la descente, gisaient tout en haut de celle-ci, tandis qu'une chambre nouvelle était aménagée pour les «esclaves» devant la tombe 417 et, à Mycènes, une fosse creusée devant la tombe de Clytemnestre. Certains corps auraient bénéficié de quelques objets alors que d'autres étaient privés de toute attention, et il s'en serait même trouvé qui auraient été écartelés, et dont quelques morceaux auraient été ensevelis négligemment dans le dromos. Il est difficile, en ces circonstances, de parler de cérémonies coutumières d'immolations humaines en faveur des défunts. La situation n'est pas différente en ce qui concerne les suttees, puisque jamais preuve n'est faite du meurtre rituel de l'épouse.

Le plus surprenant, tant en ce qui concerne l'élimination rituelle de veuves que la mise à mort de soi-disant serviteurs, reste le nombre de cas considérés comme possibles, dérisoirement faible. Or, à partir du moment où ces usages généraux font défaut, les cas isolés perdent la petite part de crédibilité qui leur restait, dans la mesure où ce genre de coutume, pour exister et survivre, me semble devoir s'inscrire au sein 
de croyances partagées par toute la population, et en conséquence mises en pratique avec un minimum de régularité. Ce chiffre apparaît davantage encore dérisoire quand on le compare au nombre, assez élevé, des tombes mycéniennes, à tholos ou à chambre, ou de tombes archaiques fouillées ${ }^{70}$ : au total, je m'accorde avec Hughes pour dire que seuls les cas de la tombe 15 de Mycènes et de Kazarma restent envisageables pour la Grèce propre, mais avec beaucoup de réserves; de la sorte, la théorie qui ferait descendre l'usage cypriote des coutumes mycéniennes se voit privée de tout crédit. Si coutume cypriote il y a, son origine doit donc être recherchée ailleurs qu'en Grèce propre. Et même les découvertes faites dans l'île du Levant ne sont pas à l'abri de la critique : restent au rang des cas vraisemblables la tombe 422 de Lapithos et la deuxième de Salamine. Il subsiste néanmoins à leur égard de nombreuses inconnues, lesquelles gagneraient à être revues dans une optique simplement funéraire, et non plus sacrificielle. Même la proximité de squelettes animaux et humains au sein d'un même dromos, en effet, ne s'est pas révélée décisive; il existe de nombreuses tombes où l'on n'a retrouvé que des ossements chevalins ou que des ossements humains : seuls deux cas associent les deux types de dépouilles, et le hasard pourrait s'en être mêlé ${ }^{71}$.

70 Olivier PELON (Tholoi [cité n. 44], passim), qui étudie uniquement tholoi et monuments circulaires, travaille sur quelque 300 sites et pour le seul âge du Bronze. Il faut y ajouter toutes les tombes à chambre, à ciste, les incinérations... sans oublier les tombeaux de l'âge du Fer, et sans compter les réutilisations très fréquentes qui augmentent de façon considérable le nombre d'inhumations attestées. Voir D. HuGHES, Human Sacrifice, p. 66-67.

71 Pour parler de deux cas, il faut accepter noir sur blanc les indications plutôt maigres à propos de la tombe de Tamassos, fouillée d'ailleurs à la fin du XIXe, à l'époque héroïque des fouilles archéologiques, quand on croyait aux sacrifices humains révélés par Tsountas, et quand les poèmes homériques jouissaient encore d'un crédit inviolable. A Kokla (Argolide), on a trouvé, au milieu du dromos et à mi-hauteur, devant la porte, les squelettes de deux chèvres, sacrifiées aux morts, ou plutôt lors du transfert de leurs cadavres, car la tombe, inviolée, avait été retrouvée vide d'ossements (K. DEMAKopoulou, The Burial Ritual in the Tholos Tomb at Kokla, Argolis, in Celebrations of Death and Divinity in the Bronze Age Argolid. Proceedings of the Sixth International Symposium at the Swedish Institute at Athens, 11-13 June, 1988, éds R. HÄGG et G.C. NoRDQUIST, Stockholm, 1990 [ActaAth, 50], p. 122-123) : une telle découverte, associée à celles de carcasses de chevaux, montre cependant que des immolations étaient possibles à l'endroit même où l'on a retrouvé parfois des squelettes humains. On en conclura pas pour autant que ces humains aient été nécessairement sacrifiés. 
Les cas de sacrifice humain dans les tombes et les dromoi reposent donc en grande partie, sinon en tout, sur l'interprétation erronée des découvertes archéologiques; il en sera ainsi tant qu'avec naïveté coupable, on considérera la simple proximité de certains squelettes suffisante à prouver qu'un des individus fût jadis sacrifié au profit des autres. De même, l'absence d'offrandes funéraires, perpétuellement constatée comme l'indice avant-coureur d'une mort sacrificielle, perd toute force dès qu'on consulte les catalogues de tombes des nécropoles, comme celui d'Asinè où, pour les tombes pré-géométriques, plus des deux-tiers des inhumations se voient assigner la mention laconique : "no burial gifts». Les concepts mêmes de maîtres et de serviteurs résistent d'ailleurs peu à l'examen, à partir du moment où les tombes concernées par les prétendus sacrifices humains sont loin d'être parmi les plus riches ou encore les plus grandes des nécropoles fouillées ${ }^{72}$.

Au total, le nombre si peu élevé de cas, égrenés du minoen moyen (ca 1700 avant J.-C.) au cypro-archaïque (ca 600 avant J.-C.), isolés parmi les milliers de sites de la civilisation du Bronze et du début de celle du Fer, et de surcroît improuvables, interdit formellement de conclure à l'existence de sacrifices humains et, a fortiori, à grande échelle.

Université Catholique de Louvain

Pierre BONNECHERE

Collège Érasme

Place Blaise Pascal, 1

B 1348 Louvain-la-Neuve

72 G.C. NORdquist (Middle Helladic Burial Rites : Some Speculations, in Celebrations of Death and Divinity in the Bronze Age Argolid [cité n. 71], p. 3738) précise d'ailleurs qu'en Argolide, lors de l'helladique moyen, les tombes les plus petites peuvent renfermer les offrandes les plus riches, et les plus grandes être totalement dépourvues de mobilier funéraire. Le sexe et l'âge n'auraient que peu à voir également avec les offrandes : il est donc vain de rechercher dans la richesse de l'inhumation d'hypothétiques rapports "maître-esclave». 